\title{
Determiner of Poor Sleep Quality in Chronic Kidney Disease Patients Links to Elevated Diastolic Blood Pressure, hs-CRP, and Blood-count-based Inflammatory Predictors
}

\author{
Maulana Antiyan Empitu1, Ika Nindya Kadariswantiningsih ${ }^{2}$, Mochammad Thaha, \\ Cahyo Wibisono Nugroho ${ }^{3}$, Eka Arum Cahyaning Putri ${ }^{4}$, Zaky El Hakim ${ }^{5}$, \\ Maulana Muhtadin Suryansyah ${ }^{5}$, Rieza Rizqi Alda ${ }^{5}$, Mohammad Yusuf Alsagaff ${ }^{6}$, \\ Mochammad Amin ${ }^{7}$, Djoko Santoso ${ }^{3}$, Yusuke Suzuki ${ }^{8}$ \\ ${ }^{1}$ Department of Pharmacology, Faculty of Medicine, Airlangga University, Jl. Mayjen Prof. Dr. Moestopo 47, Surabaya, Indonesia \\ ${ }^{2}$ Department of Medical Microbiology, Faculty of Medicine, Airlangga University, Jl. Mayjen Prof. Dr. Moestopo 47, Surabaya, Indonesia \\ ${ }^{3}$ Department of Internal Medicine, Faculty of Medicine, Airlangga University, Jl. Mayjen Prof. Dr. Moestopo 47, Surabaya, Indonesia \\ ${ }^{4}$ Department of Physiology, Faculty of Medicine, Airlangga University, Jl. Mayjen Prof. Dr. Moestopo 47, Surabaya, Indonesia \\ ${ }^{5}$ Medical Science Program, Faculty of Medicine, Airlangga University, Jl. Mayjen Prof. Dr. Moestopo 47, Surabaya, Indonesia \\ ${ }^{6}$ Department of Cardiology, Faculty of Medicine, Airlangga University, Jl. Mayjen Prof. Dr. Moestopo 47, Surabaya, Indonesia \\ ${ }^{7}$ Institute of Tropical Disease, Airlangga University, Jl. Mayjen Prof. Dr. Moestopo 47, Surabaya, Indonesia \\ ${ }^{8}$ Division of Nephrology, Juntendo University School of Medicine, 2-1-1, Hongo, Bunkyo-ku, Tokyo, Japan \\ *Corresponding author. E-mail: mochthaha@yahoo.com
}

Received date: Oct 20, 2017; Revised date: Dec 7, 2018; Accepted date: Dec 10, 2018

\section{Abstract}

B

ACKGROUND: Sleep deprivation is strongly associated with cardiovascular disease (CVD) via sympathetic overstimulation and systemic inflammation in general population. However, the significance of poor sleep quality in chronic kidney disease (CKD) is still underexplored.

METHODS: This study assessed the sleep quality of 39 with non-dialysis CKD (ND CKD) patients and 25 hemodialysis CKD (HD CKD) patients using the Pittsburgh Sleep Quality Index (PSQI) questionnaire. Poor sleeper was defined as individual with PSQI $>5$.

RESULTS: The prevalence of poor sleeper (30\% vs. $60 \%$, $p=0.029$ ) and the cummulative PSQI (ND CKD 4.5 $\pm 4.4, \mathrm{HD}$ CKD $8 \pm 6, p=0.038$ ) are different between ND CKD and HD CKD groups. Among the ND CKD, there are association between short sleep duration $(<5$ hours per day) with elevated diastolic blood pressure groups ( $\mathrm{r}=0.421, p<0.05$ ); habitual sleep efficiency with platelet-to-lymphocyte ratio $(\mathrm{r}=0.532, p<0.0001)$; daytime dysfunction with increased hs-CRP ( $\mathrm{r}=0.345, p=0.032)$ and neutrophil-to-lymphocyte ratio $(\mathrm{r}=0.320, p=0.046)$. In HD CKD group, a requirement to use sleep medication was associated with elevated highsensitivity C-reactive protein (hs-CRP) level ( $\mathrm{r}=0.434$, $p=0.030)$ and decreased monocyte-to-lymphocyte ratio ( $\mathrm{r}=$ $0.410, p=0.042$ ); daytime dysfunction was associated with serum hs-CRP $(\mathrm{r}=0.452, p=0.023)$.

CONCLUSION: This study revealed that some features of poor sleep quality in CKD patients including low sleep efficiency, daytime dysfunction and requirement to use sleep medication were associated with increased diastolic blood pressure, hs-CRP and blood-count-based inflammatory predictors. Thus, this finding prompt to pay closer attention to sleep complaints in the management of CVD risk factors in CKD patients.

KEYWORDS: sleep quality, chronic kidney disease, blood pressure, inflammation

Indones Biomed J. 2019; 11(1): 100-6 


\section{Introduction}

Chronic kidney disease (CKD) is a progressive disease with high incidence and mortality rate. $(1,2)$ The decreased kidney function in CKD stems from the progressive glomerular damage that is caused by the maladaptive response of functional kidney resident cells toward various stress including inflammation, oxidative stress and hypertension. $(3,4)$ The mortality risk of an individual with CKD is increased along with CKD progressivity and strongly associated with cardiovascular risk factors such as inflammation and stress oxidative.(5)

Sleep deprivation is a commonly observed condition which may associate with cardiovascular disease.(6) Sleep deprivation is experienced by approximately $15-25 \%$ of USA population and related to diastolic ventricular dysfunction, hypertension, coronary heart disease as well as conditions requiring heart-pacing.(6) The mechanisms in which sleep deprivation associated with cardiovascular disease (CVD) are sympathetic overstimulation and systemic inflammation.

During normal sleep, markedly different brain activity oscillates between two stages known rapid eye movement (REM) sleep and non-REM (NREM) sleep. During the NREM sleep, decreased sympathetic activity and activated vagal tone are responsible for the nocturnal dipping of blood pressure associated with sleep.(7) Sleep loss and sleep disorders lead to sympathetic nervous system stimulation and attenuation of the sleep-induced decrease in blood pressure.(7) Increased plasma renin activity has been observed in the non-CKD population with sleep deprivation and directly affected blood pressure by triggering vasoconstriction.(8)

Furthermore, increased plasma level of proinflammatory cytokines has also been found in individuals with sleep deprivation and sleep fragmentation.(9) Elevated plasma level of pro-inflammatory cytokines such as C-reactive protein (CRP) and interleukin (IL)-6 were associated with endothelial dysfunction and atherosclerotic disease.(10) Although numerous studies have reported the association between poor sleep quality and CVD in the general population, the interaction of sleep quality and CVD risk factors among individuals with CKD is still underexplored.

Since sleep deprivation in general population correlates well with increased risk of CVD (6), it is important to study whether sleep quality also affects the CVD risk factors among CKD population. This study evaluated components that determine the quality of sleep in both hemodialysis CKD (HD CKD) and non-dialysis CKD (ND CKD) patients using commonly used questionnaire Pittsburgh Sleep Quality Index (PSQI). The questionnaire evaluates several sleep quality components including subjective sleep quality, sleep latency, sleep duration, habitual sleep efficiency, sleep disturbances, use of sleeping medications and daytime dysfunction. This study aimed to investigate the association of those determiners of sleep quality with inflammation marker high sensitivity CRP (hs-CRP) and other bloodcount-based inflammatory predictors which correlated well with cardiovascular outcomes, such as lymphocyteto-monocyte, platelet-to-lymphocyte and neutrophilto-lymphocyte ratios.(11) The study also investigated the association between sleep quality components with blood pressure and obesity as a factor that modulates the occurrence of sleep disturbance.

\section{Methods}

\section{Study Design and Participant}

This investigation is part of Airlangga University's study in validating score-based assessment tools for malnutrition, inflammation and oxidative stress. This study is an observational study with a cross-sectional design, constitutively enrolled stage II to V CKD patients who visited a government and a private hospital in Surabaya, Indonesia on May to August 2017. The study includes 64 participants agreed and gave their consent for sleep quality assessment. The diagnosis of CKD was confirmed by The National Kidney Foundation Kidney Disease Outcomes Quality Initiative (NKF K-DOQI) criteria based on Chronic Kidney Disease Epidemiology Collaboration (CKD-EPI) equation (estimated glomerular filtration rate (eGFR) $\leq 60$ $\mathrm{mL} / \mathrm{min} / 1.73 \mathrm{~m}^{2}$, urine albumin $>300 \mathrm{mg} /$ day, Albumin to Creatinine Ratio $>250 \mathrm{mg} / \mathrm{g}$ for men and $>355 \mathrm{mg} / \mathrm{g}$ for women). The subjects who had an overt infection, malignancy, and fever in the last three days were excluded from this study. The study was approved by Airlangga University Hospital Ethics Committee (Approval number: 093/IGH/2017) and conducted by a research team with Good Clinical Practice certification.

\section{Sleep Quality Assessment}

In this study, the trained examiners interviewed the study participants based on the PSQI questionnaire as previously explained.(12) The PSQI questionnaire is an instrument to assess the sleep quality by measuring seven areas or 
components: subjective sleep quality, sleep latency, sleep duration, habitual sleep efficiency, sleep disturbances, use of sleeping medications and daytime dysfunction over the last month. This instrument can distinguish between "poor sleeper" and good sleeper". Poor sleeper was defined as PSQI Score $>5$.

\section{Laboratory Examination}

Participants underwent blood and urine sampling early in the morning. Serum creatinine, serum cystatin-C, and complete blood count were measured based on established laboratory method previously explained. This study used hs-CRP for the inflammatory biomarker. The hs-CRP is measured in serum using enzyme-linked immunosorbent assay (Roche Diagnostic, CA, USA).

\section{Anthropometry and Blood Pressure Measurement}

Anthropometric examination in this study was done to obtain a data of body weight, neck circumference, body mass index (BMI) and body fat percentage (BFP). Body weight was measured to the nearest $0.1 \mathrm{~kg}$ in balance-beam scale. The BFP was measured and calculated as described in the previous study.(13)

All blood pressure measurement was performed by trained examiner utilizing a standard stethoscope and sphygmomanometer. Resting blood pressure was measured in a situated position by auscultation utilizing the RivaRocci-Korotkoff technique following 5 minutes of rest. Systolic and diastolic blood pressure were controlled by the beginning and vanishing of the Korotkoff sounds (K1 and
K5), individually. Proper cuff sizes were chosen by the upper right arm boundary by alluding to procedural guidelines. Three BP readings were taken at 2-minute interims. The least measurement result was utilized in the analysis.

\section{Statistical Analysis}

All quantitative data were subject to normality test using the Saphiro-Wilk method. Normally distributed values were described as mean and standard deviation (SD) and not normally distributed values were stated as median (interquartile range). Difference between groups of normally distributed interval data was assessed using unpaired T-test, while the Mann-Whitney test was used in not-normally distributed interval data. The difference of proportion between groups in this study is compared using the Chisquare test. All comparison test utilized in this study used two-tailed 95\% confidence onterval. A significant difference was defined if the $p<0.05$. Data analysis is supported by XLSTAT version 2016.02.28451.

\section{Results}

\section{Characteristics of Study Participants}

Among 64 participants, 39 of them were ND CKD patients and 25 participants were HD CKD patients. There were significant differences in the proportion of poor sleepers $(30 \%$ in ND CKD, $60 \%$ in HD CKD, $p=0.029$, Table 1) as well as cumulative PSQI (ND CKD $4.5 \pm 4.4$, HD CKD $8 \pm 6, p=0.038$, Figure 1) between the twogroups.

Table 1. Characteristics of study participants.

\begin{tabular}{lccc}
\hline \multicolumn{1}{c}{ Characteristic } & $\begin{array}{c}\text { ND CKD } \\
(\mathbf{n = 3 9 )}\end{array}$ & $\begin{array}{c}\text { HD CKD } \\
(\mathbf{n = 2 5})\end{array}$ & $\boldsymbol{p}$-value \\
\hline Age (years) & $58.6 \pm 5.3$ & $57.8 \pm 10.9$ & $\mathrm{NS}$ \\
Sex ratio (F/M) & $14 / 25$ & $12 / 13$ & $\mathrm{NS}$ \\
eGFR (mL/minute/1.73 m²) & $42 \pm 26$ & $3 \pm 1$ & $<0.0001$ \\
Urine ACR & $184(12-990)$ & $1783(715-3548)$ & $<0.0001$ \\
Serum creatinine (mg/dL) & $1.8(1.2-2.5)$ & $14.0(11.2-15.9)$ & $<0.0001$ \\
Serum cystatin-C (mg/L) & $1.7(1.2-2.6)$ & $7.5(6.5-7.9)$ & $<0.0001$ \\
hs-CRP (mg/L) & $2.8(0.4-13.9)$ & $6.7(0.6-36)$ & 0.005 \\
Poor sleeper, $\mathrm{n}(\%)$ & $12(30)$ & $15(60)$ & 0.029 \\
Body weight $(\mathrm{kg})$ & $70.3 \pm 12.5$ & $64.2 \pm 14.9$ & 0.045 \\
BMI & $26 . \pm 4.2$ & $25.1 \pm 4.5$ & $\mathrm{NS}$ \\
BFP (\%) & $20.9 \pm 7.5$ & $19.0 \pm 7.8$ & $\mathrm{NS}$ \\
\hline
\end{tabular}

Data are expressed in Mean $\pm \mathrm{SD}$ or Median (Interquartile range). $p<0.05$ was considered significant. eGFR: estimated glomerular filtration rate; ACR: albumin to creatinine ratio; hs-CRP: high sensitivity $\mathrm{C}$-reactive protein; BMI: body mass index; BFP: Body fat percentage; NS: not significant. 


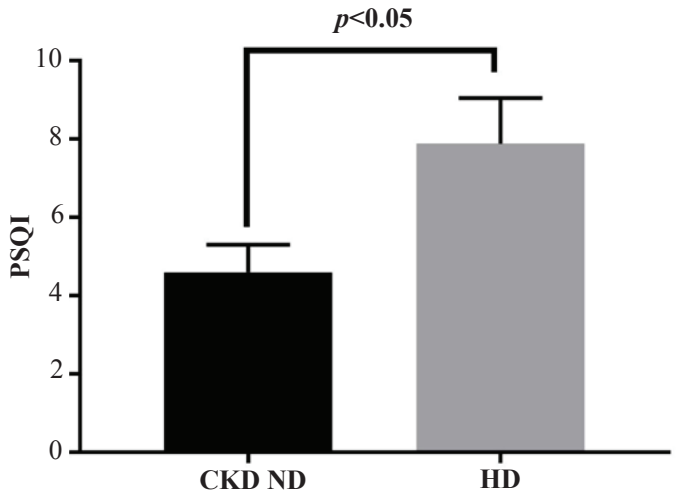

Figure 1. Comparison of cummulative sleep quality index between NDCKD and HD CKD groups.

\section{Association of Sleep Duration and Blood Pressure}

As the sympathetic activity is reduced and the vagal tone is elevated during the NREM sleep (7), this study examined whether the average sleep duration per day is associated with the reduction of blood pressure. This study found that the short sleep duration (less than 5 hours per day) is associated with elevated diastolic blood pressure among the ND CKD groups $(\mathrm{r}=0.421, p<0.05)$. There is an observed trend that the diastolic blood pressure is higher in individuals with shorter daily sleep duration, despite the insignificant statistical difference (Figure 2). In contrast, there is no association of daily sleep duration with diastolic blood pressure in the HD CKD group.

\section{Association of Habitual Sleep Efficiency and Platelet-to- lymphocyte Ratio}

Habitual sleep efficiency (HBE) is an estimate of effective sleep time versus the time spent of getting sleep. This

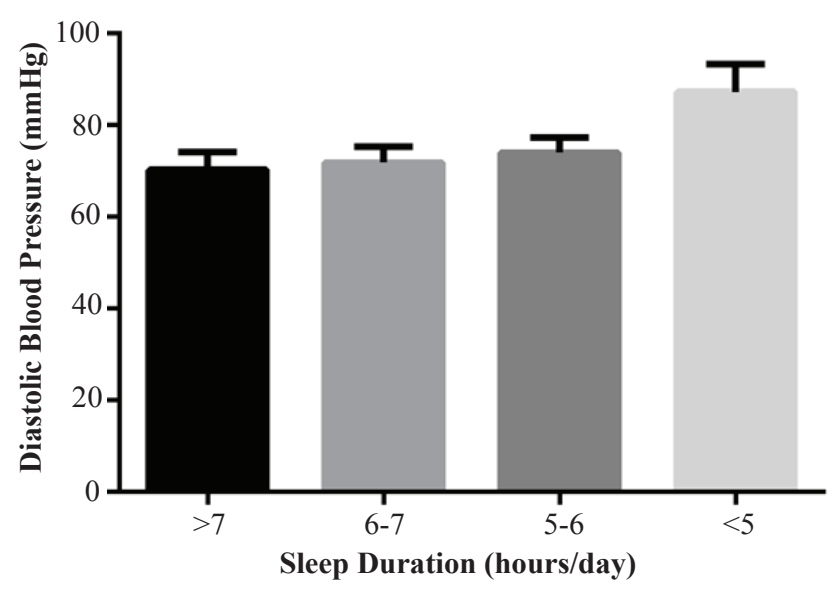

Figure 2. Comparison of diastolic blood pressure among ND CKD patients based on the daily sleep duration. ratio represents the actual sleep instead of the time spent in bed during 24 hours. To know whether the time spent on effective sleep is associated with the predictor of vascular outcome, this study investigated the association of between the HBE and platelet-to-lymphocyte ratio. In ND CKD group, habitual sleep efficiency was positively associated with platelet-to-lymphocyte ratio $(\mathrm{r}=0.532, p<0.0001)$.

\section{Association between the Severity of Sleep Disturbance with Serum hs-CRP level and Monocyte-to-lymphocyte Ratio}

A requirement to use sleep medication and whether the sleep disturbance deteriorates the daily function are among the determiner of sleep disturbance severity. To assess whether the severity of sleep disturbance affects the inflammatory states or vice versa, this study investigated the association between the requirement to use sleep medication and daily dysfunction with inflammatory markers. In HD group, a requirement to use sleep medication was negatively correlated with serum hs-CRP level $(\mathrm{r}=0.434, p=0.030)$ and monocyte-to-lymphocyte ratio $(\mathrm{r}=-0.410, p=0.042)$. However, a requirement to use sleep medication was not correlated with serum hs-CRP level neither mono-tolymphocyte ratio in ND CKD group. A daytime dysfunction was associated with serum hs-CRP level in both ND CKD ( $\mathrm{r}=0.345, p=0.032)$ and HD CKD groups $(\mathrm{r}=0.452$, $p=0.023$, Table 2 ). Neutrophil-to-lymphocyte ratio was also correlated with daytime dysfunction in ND CKD group ( $\mathrm{r}=0.320, p=0.046$, Table 2).

\section{Association of Anthropometric Measurement with Sleep Disturbance}

As the obstructive sleep apnea (OSA) is prevalent among individuals with obesity (14), this study examined whether higher PSQI score which represents poor sleep quality is associated with anthropometric features of obesity. We that bigger neck circumference is associated with sleep disturbance component of PSQI score among the ND CKD group $(\mathrm{r}=0.391, p=0.01)$. This study also showed a trend that participants with PSQI score $>5$ has a higher BFP compared to participants with PSQI score of less than 5, despite the statistically non-significant different (Figure 3).

\section{Discussion}

This study showed that the poor sleepers were more prevalent among the HD CKD compared to the ND CKD group. The higher cumulative score of PSQI which represents poorer 
Table 2. Association between daytime dysfunction with hs-CRP level and neutrophil-to-lymphocyte ratio

\begin{tabular}{lccccc}
\hline \multirow{2}{*}{ Characteristic } & \multicolumn{2}{c}{ ND CKD } & \multicolumn{2}{c}{ HD CKD } \\
\cline { 2 - 5 } & $\mathbf{r}$ & $\boldsymbol{p}$-value & r & $\boldsymbol{p}$-value \\
\hline Serum hs-CRP & 0.345 & 0.032 & 0.452 & 0.023 \\
Neutrophil-to-lymphocyte ratio & 0.32 & 0.046 & - & NS \\
\hline
\end{tabular}

hs-CRP: high sensitivity C-reactive protein; NS: not significant.

sleep quality was also found among participants in HD CKD compared to ND CKD patients. This result confirmed the previous finding that sleep disorder is more common in endstage renal disease (ESRD) patients receiving hemodialysis therapy compared to patients with a mild decrease in kidney function.(15) The previously reported prevalence of poor sleep quality assessed using PSQI in hemodialysis patients ranges between $34-78 \%$ (16), which is in accordance with the prevalence in this study.

The deteriorating effect of sleep disturbance in aggravating cardiovascular risk may stem from its influence in blood pressure. In the Coronary Artery Risk Development in Young Adults (CARDIA) Sleep Study, the shorter sleep duration and low sleep maintenance associated with poor control of both systolic and diastolic blood pressure after 5 years.(17) In this study, the short sleep duration was associated with higher diastolic blood pressure. There is also an observed trend that ND CKD patients with less than 5 hours of sleep per day have higher diastolic blood pressure. The oscillation between REM and NREM sleep drive the oscillation in plasma renin activity (PRA).(18) During the NREM sleep, both systolic and diastolic baseline decrease due to decreased PRA and increased vagal tone. A study in ESRD patients revealed that the hemodialysis patients failed to increase the cardiac vagal tone during the NREM sleep (19).

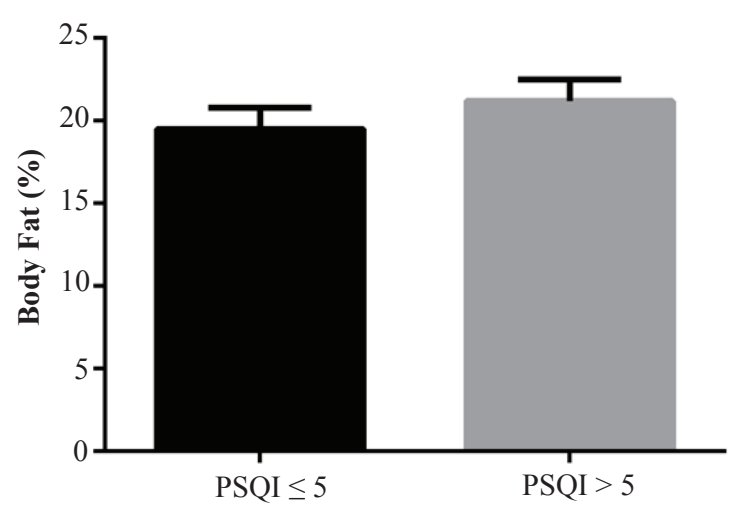

Figure 3. BFP comparison of CKD patients with PSQI score $>5$ and $\leq 5$.
The link between poor sleep quality and elevated blood pressure in this study may also be linked to inflammation. The habitual sleep efficiency in this study was associated with platelet-to-lymphocyte ratio, a predictor of atherosclerosis and complicated hypertension.(20) The severity of sleep disturbance also associated with alteration of other markers of inflammation such as lymphocyte-to-monocyte and neutrophil-to-lymphocyte ratio. Although how the immune system participates in the regulation of sleep is not fully understood, several clues indicate that different kind of cytokines affects sleep architecture (21). IL-1 and tumor necrosis factors (TNF) affect the acquisition of NREM sleep across different species including rat, mouse, monkey, cat, rabbit, and sheep regardless of the administration route. $(21,22)$ Immune-reactive neurons for IL-1 and TNF in the hypothalamus, the hippocampus, and the brainstem are implicated in the regulation of sleep-wake behavior. Stimulation by TNF, IL-1, or IL-6 regulates the release of nitric oxide, serotonin and adenosine which tightly regulate sleep-wake cycle.(23) Since declining kidney function associated with chronic inflammation and oxidative stress $(4,24)$, it is likely that dysregulation of cytokine secretion by immune system components in CKD alter the sleep-wake architecture and sleep quality.

A previous study also showed that that poor sleep quality was correlated with higher inflammatory markers such as hs-CRP (25). Our study did not demonstrate the association of cumulative PSQI with increased serum hsCRP level, which might be due to the small sample. However, this study showed that hs-CRP level was associated with two components of PSQI which describe the severity of sleep disorders: a requirement to use medication and daytime dysfunction.

In the previous study, obesity was found among the most common risk for OSA.(14) This study showed the positive association between the sleep disturbance score of PSQI with neck circumference, accompanied by a trend of higher body fat percentage among participants with poor sleep characteristic. Among the characteristic of sleep disturbance that was documented using PSQI 
questionnaire in this study is relevant to the symptom of OSA, including the uncomfortable breath, loud snore, and suddenly waking up in the middle of the sleep. This result indicates that the sleep disturbance among this study participant might be partly related to obesity. Additionally, this finding strengthens the established understanding about the negative impact of obesity along with increased risk for CVD, chronic inflammation, and type 2 diabetes mellitus. $(13,26)$

In viewing this result, several limitations such as the cross-sectional nature of this study and small size should be considered. The use of PSQI as an instrument of sleep assessment may result in widely different estimates of sleep disorders. However, the prevalence of poor sleeper in this study fell within the range of previous studies using PSQI as their sleep assessment instruments. $(27,28)$

\section{Conclusion}

In summary, our study confirmed the higher prevalence of poor sleeper among HD CKD compared to ND CKD patients and demonstrated the association of characteristics of poor sleep quality with increased inflammatory markers. Thus, this result prompts practicing nephrologist to pay closer attention to sleep complaints in managing CVD risk factors in CKD.

\section{References}

1. Levin A, Tonelli M, Bonventre J, Coresh J, Donner JA, Fogo AB, et al. Global kidney health 2017 and beyond: a roadmap for closing gaps in care, research, and policy. Lancet. 2017; 390: 1888-917

2. Jha V, Garcia-Garcia G, Iseki K, Li Z, Naicker S, Plattner B, et al. Chronic kidney disease: global dimension and perspectives. Lancet. 2013; 382: 260-72.

3. Empitu MA, Kadariswantiningsih IN, Aizawa M, Asanuma K. MAGI-2 and scaffold proteins in glomerulopathy. Am J Physiol Physiol. 2018; 315: F1336-44.

4. Thaha M, Yusuf M, Empitu MA, Bakarman A, Tomino Y. Distribution of dimethylarginine-dimethylaminohydrolase-II (DDAH2) gene polymorphism in hemodialysis patients. Acta Med Indones. 2013; 45: 83-8.

5. Kasiske BL, Wheeler DC. KDIGO clinical practice guideline for the evaluation and management of chronic kidney disease: Foreword. Kidney Int Suppl. 2013; 3(1): 2. doi:10.1038/kisup.2012.74.

6. Turek NF, Ricardo AC, Lash JP. Sleep disturbances as nontraditional risk factors for development and progression of CKD: review of the evidence. Am J Kidney Dis. 2012; 60: 823-33.

7. Spiegel K, Leproult R, L'Hermite-Balériaux M, Copinschi G, Penev PD, Van Cauter E. Leptin levels are dependent on sleep duration: relationships with sympathovagal balance, carbohydrate regulation, cortisol, and thyrotropin. J Clin Endocrinol Metab. 2004;89(11):5762-71.

8. Murck H, Uhr M, Ziegenbein M, Künzel H, Held K, Antonijevic IA, et al. Renin-angiotensin-aldosterone system, HPA-axis and sleepEEG changes in unmedicated patients with depression after total sleep deprivation. Pharmacopsychiatry. 2006; 39: 23-9.

9. Patel SR, Zhu X, Storfer-Isser A, Mehra R, Jenny NS, Tracy R, et al. Sleep duration and biomarkers of inflammation. Sleep. 2009; 32: 200-4.

10. Xu G, Luo K, Liu H, Huang T, Fang X, Tu W. The progress of inflammation and oxidative stress in patients with chronic kidney disease. Renal Failure. 2015; 37: 45-9.

11. Malhotra R, Marcelli D, von Gersdorff G, Grassmann A, Schaller $\mathrm{M}$, Bayh I, et al. Relationship of neutrophil-to-lymphocyte ratio and serum albumin levels with C-reactive protein in hemodialysis patients: results from 2 international cohort studies. Nephron. 2015; 130: 263-70.

12. Buysse DJ, Reynolds III CF, Monk TH, Berman SR, Kupfer DJ. The Pittsburgh Sleep Quality Index: a new instrument for psychiatric practice and research. Psychiatry Res. 1989; 28: 19-213.

13. Thaha M, Empitu MA, Kadariswantiningsih IN, Nugroho CW, Hasanatuludhhiyah N, Rasyid H, et al. Anthropometry-based body fat percentage predicts high hs-CRP in chronic kidney disease patients. Indones Biomed J. 2018; 10: 184-91.

14. Wolk R, Somers VK. Obesity-related cardiovascular disease: implications of obstructive sleep apnea. Diabetes, Obes Metab. 2006; 8: 250-60.

15. Merlino G, Piani A, Dolso P, Adorati M, Cancelli I, Valente M, et al. Sleep disorders in patients with end-stage renal disease undergoing dialysis therapy. Nephrol Dial Transplant. 2005; 21: 184-90.

16. Nishiyama T, Mizuno T, Kojima M, Suzuki S, Kitajima T, Ando KB, et al. Criterion validity of the Pittsburgh Sleep Quality Index and Epworth Sleepiness Scale for the diagnosis of sleep disorders. Sleep Med. 2014; 15: 422-9.

17. Knutson KL, Van Cauter E, Rathouz PJ, Yan LL, Hulley SB, Liu K, et al. Association between sleep and blood pressure in midlife: the CARDIA sleep study. Arch Intern Med. 2009; 169: 1055-61.

18. Brandenberger G, Ehrhart J, Piquard F, Simon C. Inverse coupling between ultradian oscillations in delta wave activity and heart rate variability during sleep. Clin Neurophysiol. 2001; 112: 992-6.

19. Roumelioti M-E, Ranpuria R, Hall M, Hotchkiss JR, Chan CT, Unruh $\mathrm{ML}$, et al. Abnormal nocturnal heart rate variability response among chronic kidney disease and dialysis patients during wakefulness and sleep. Nephrol Dial Transplant. 2010; 25: 3733-41.

20. Turkmen K, Erdur FM, Ozcicek F, Ozcicek A, Akbas EM, Ozbicer A, et al. Platelet-to-lymphocyte ratio better predicts inflammation than neutrophil-to-lymphocyte ratio in end-stage renal disease patients. Hemodial Int. 2013; 17: 391-6.

21. Imeri L, Opp MR. How (and why) the immune system makes us sleep. Nat Rev Neurosci. 2009; 10: 199-210.

22. Opp MR. Cytokines and sleep. Sleep Med Rev. 2005; 9: 355-64.

23. Krueger JM. The role of cytokines in sleep regulation. Curr Pharm Des. 2008; 14: 3408-16.

24. Stenvinkel P, Gillespie IA, Tunks J, Addison J, Kronenberg F, Drueke $\mathrm{TB}$, et al. Inflammation modifies the paradoxical association between body mass index and mortality in hemodialysis patients. J Am Soc Nephrol. 2016; 27: 1479-86.

25. Zhang J, Wang C, Gong W, Peng H, Tang Y, Li CC, et al. Association between sleep quality and cardiovascular damage in pre-dialysis patients with chronic kidney disease. BMC Nephrol. 2014; 15: 131. doi: 10.1186/1471-2369-15-131. 
26. Mokdad AH, Ford ES, Bowman BA, Dietz WH, Vinicor F, Bales VS, et al. Prevalence of obesity, diabetes, and obesity-related health risk factors, 2001. Jama. 2003; 289: 76-9.

27. Plantinga L, Lee K, Inker LA, Saran R, Yee J, Gillespie B, et al.
Association of sleep-related problems with CKD in the United States, 2005-2008. Am J Kidney Dis. 2011; 58: 554-64.

28. Santo De RM, Bartiromo M, Cesare CM, Cirillo M. Sleep disorders occur very early in chronic kidney disease. J Nephrol. 2008; 21: S59-65. 